\title{
Successful repair of an ascending aorta injured by a displaced sternal plate
}

\author{
Igor Vendramin, MD, ${ }^{\mathrm{a}}$ Paolo Frigatti, MD, ${ }^{\mathrm{b}}$ Daniela Piani, MD, ${ }^{\mathrm{a}}$ Massimo Sponza, MD, \\ Uberto Bortolotti, MD, ${ }^{\mathrm{a}}$ and Ugolino Livi, $\mathrm{MD},{ }^{\mathrm{a}}$ Udine, Italy
}

\author{
From the ${ }^{\mathrm{a} C}$ Cardiothoracic Department, ${ }^{\mathrm{b}}$ Vascular Division, Department of Surgery, and ${ }^{\mathrm{c}}$ Interventional Division, \\ Department of Radiology, University Hospital of Udine, Udine, Italy. \\ Disclosures: Authors have nothing to disclose with regard to commercial support. \\ Received for publication June 11, 2019; revisions received July 27, 2019; accepted for publication July 29, 2019; \\ available ahead of print Oct 8, 2019. \\ Address for reprints: Igor Vendramin, MD, Cardiothoracic Department, P. le S.M. Misericordia 15, Udine, Italy \\ (E-mail: vendramin.igor@gmail.com). \\ J Thorac Cardiovasc Surg 2020;159:e151-4 \\ $0022-5223 / \$ 36.00$ \\ Copyright (C) 2019 by The American Association for Thoracic Surgery \\ https://doi.org/10.1016/j.jtcvs.2019.07.128
}

Video clip is available online.

Malignant neoplasms involving the sternum are uncommon, with primary chondrosarcoma (PC) the most frequent. ${ }^{1}$ Treatment includes radical sternal resection followed by reconstruction using various prosthetic or biological materials, associated with musculocutaneous flaps. ${ }^{2}$ Complications after sternal reconstruction are rare and mainly include wound and flap infection and rupture of titanium bars. ${ }^{2}$ We describe a patient in whom a rigid sternal plate used for sternal reconstruction for PC caused a rupture of the ascending aorta. This previously unreported complication was successfully managed with a multidisciplinary approach.

\section{CLINICAL SUMMARY}

A 52-year-old man was referred to our unit because of a pulsatile sternal mass. Eight years earlier he had undergone total sternal excision because of PC and replacement with a Marlex mesh plate and latissimus dorsi muscle flap. At 6 months before admission, a computed tomography (CT) scan was normal. An emergency CT scan revealed a $9 \times 5$ - mm pseudoaneurysm due to erosion by the collapsed sternal plate of the ascending aorta at the origin of the innominate artery infiltrating the musculocutaneous plane (Figure 1), as well as a bovine aortic arch. A 2D echocardiogram showed normal left ventricular function, no pulmonary hypertension, and no valvular disease.

The initial surgical approach consisted in debranching of the brachiocephalic vessels, through a left posterolateral

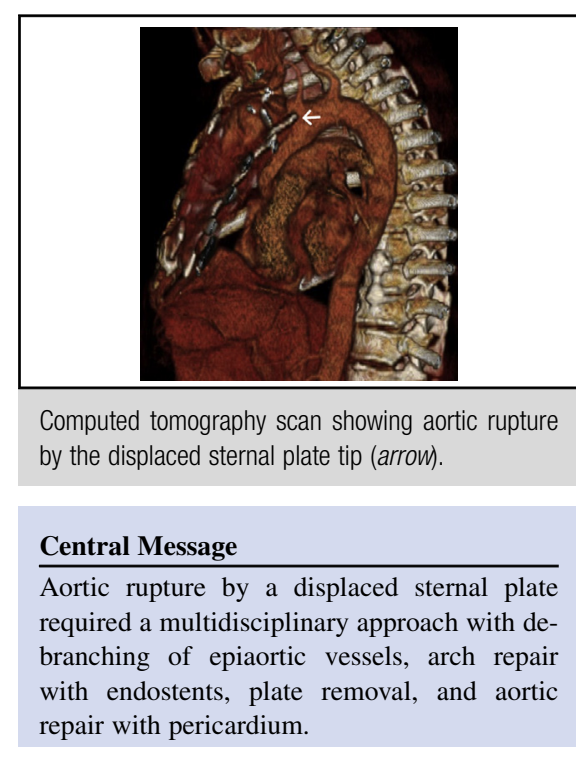

See Commentary on page e155.

thoracotomy, using a 12-mm bifurcated graft (Vascutek, Inchinnan, Scotland) sutured to the descending aorta distally and to the left subclavian and left carotid arteries with two 8-mm branches proximally, followed by a carotid-to-carotid bypass with an $8-\mathrm{mm}$ graft. Two days later, the origin of the innominate artery was closed with an 18-mm Amplatzer device (Abbott Laboratories, Chicago, Ill), and a 46/44 × 100-mm endograft (Bolton Medical, Sunrise, Fla) was inserted into the aortic arch to cover the origin of the pseudoaneurysm (Figure 2, A). The control CT scan showed patency of the brachiocephalic vessels, partial compression of the endostent by the sternal plate, and no endoleaks (Figure 2, $B$, and Video 1).

One month later, another CT scan showed a type I endoleak and progression of the sternal compression, necessitating insertion of a second endograft. Subsequently, the sternal plate was explanted, and all thrombotic and necrotic material was removed. The aortic lesion was closed with a pericardial patch, and final chest reconstruction was achieved by interposition of the omentum under a titanium mesh and wound closure using the previous latissimus dorsi flap after adequate mobilization. Cultures of the excised material grew Staphylococcus epidermidis. A 6-month 

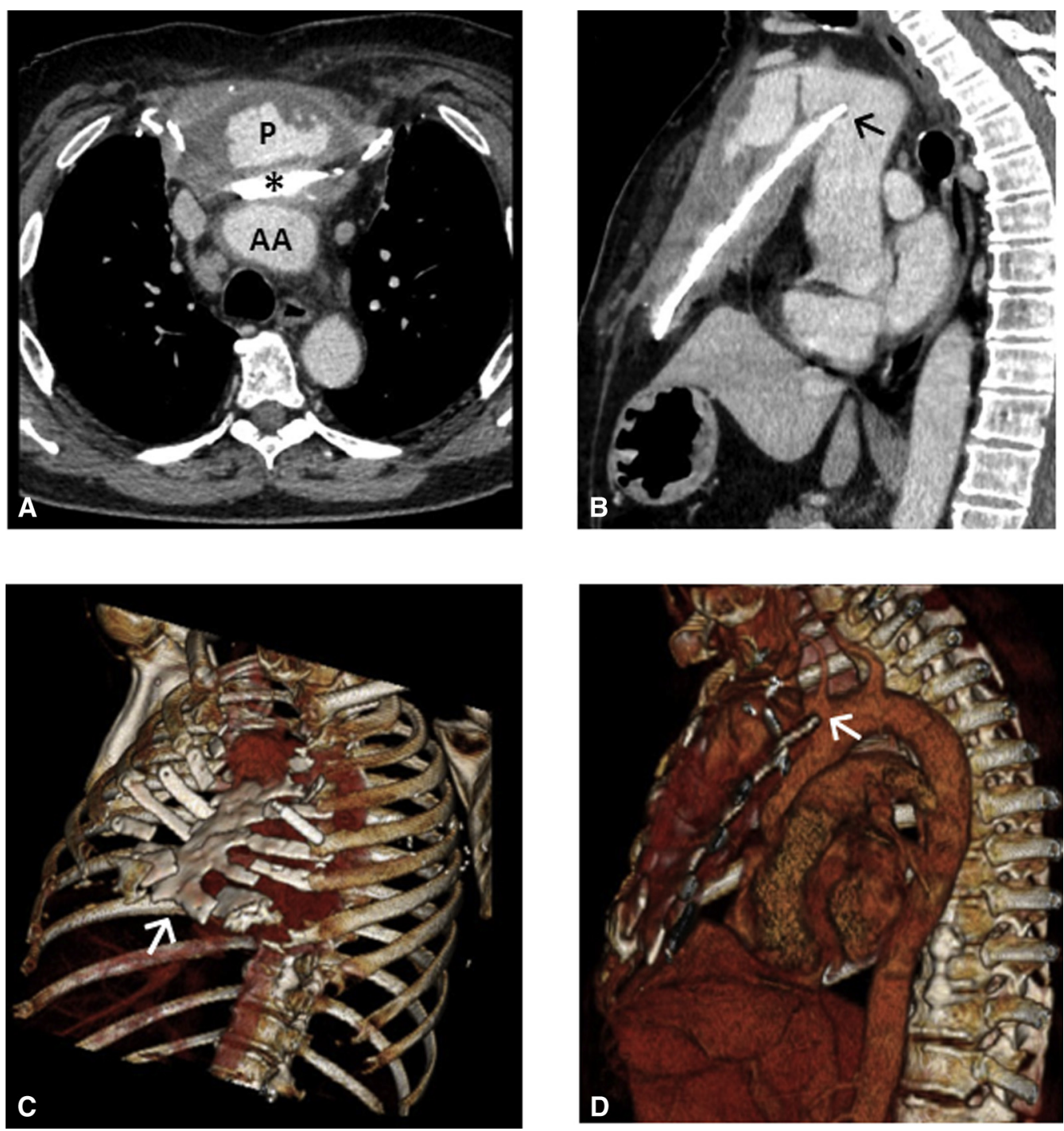

FIGURE 1. A, Computed tomography scan showing the sternal plate $(*)$ with pseudoaneurysm $(P)$ formation from the ascending aorta $(A A)$. B, The distal edge of the plate (arrow). C and D, Impingements in the distal aorta shown on the 3D reconstructions (arrows).

control CT scan demonstrated stability of the repair (Figure 2, $C$ and $D$ ).

\section{DISCUSSION}

Sternal PC is a rare neoplasm that requires radical excision and reconstruction of the anterior chest wall. A successful procedure can prevent respiratory failure due to paradoxical chest movements, avoid mediastinal infections, and protect the underlying structures. ${ }^{2,3}$ Various prosthetic materials have been used to reconstruct the sternum excised because of primary or metastatic neoplasms, including methyl methacrylate, polytetrafluoroethylene, titanium, and, more recently, bioceramics. ${ }^{2-5}$

Sternal replacement is associated with a low incidence of periprocedural and late complications, which include mainly wound instability and infection. However, experience with sternal reconstruction is usually confined to single case reports, and thus many unusual complications may be underreported. The present patient presented with an extremely complex and previously unreported complication following sternal replacement for PC 8 years earlier. Aortic rupture was caused by aortic wall injury due to the displaced sternal plate; in the absence of major thoracic trauma, it may be speculated that this might have been a consequence of the progressive weakening of the costal attachments secondary to the mechanical effects of continuous breathing movements. In the absence of abscess, the underlying infection was considered to be superimposed on the retrosternal blood collection from the ruptured aorta. 

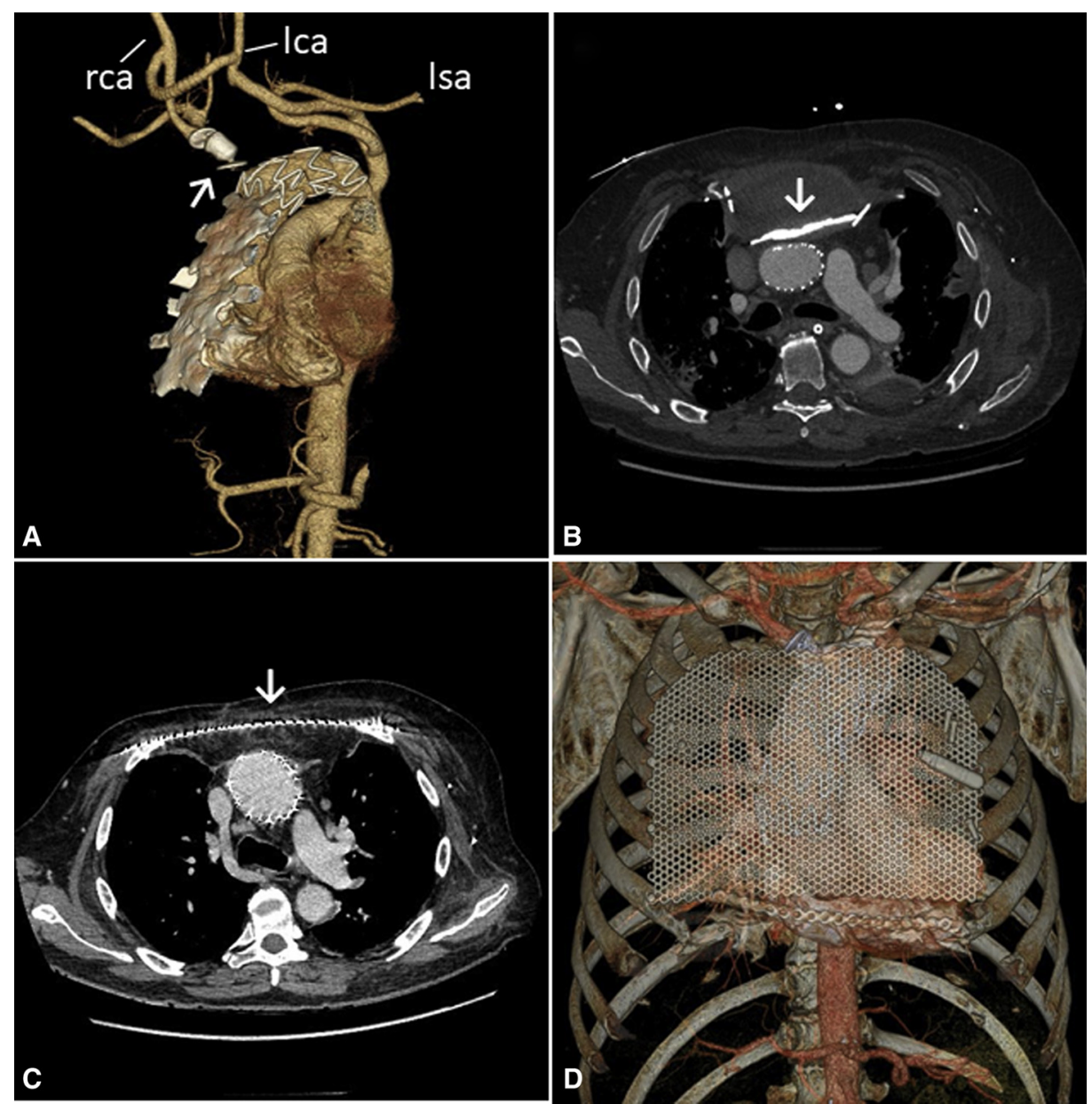

FIGURE 2. A, 3D reconstruction showing debranching of the left subclavian artery and left carotid artery $(L C A)$ with an LCA-to-right carotid artery $(R C A)$ bypass, and the Amplatzer device (arrow) occluding the origin of the innominate artery. B, The plate compressing the endograft (arrow). C and D, Final result with the new sternal reconstruction (arrow).

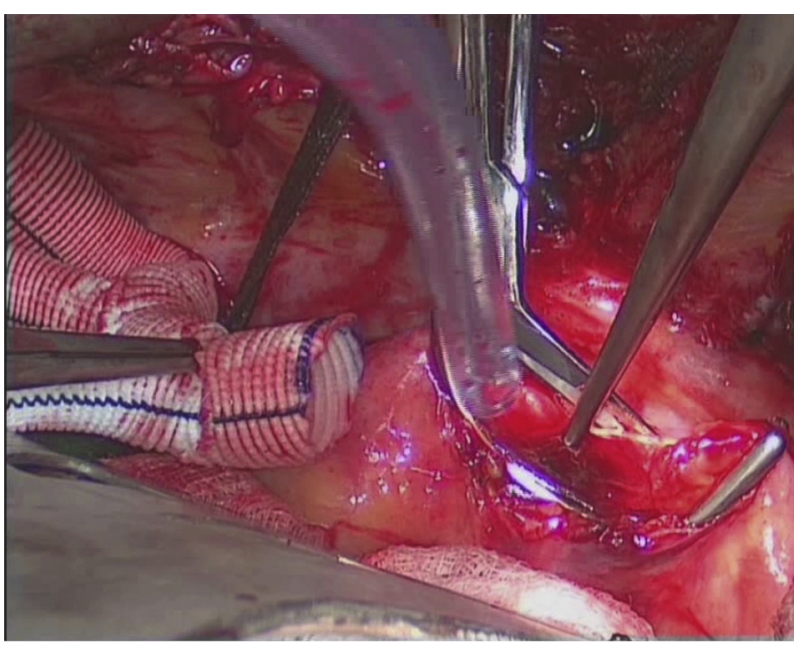

VIDEO 1. Successful repair of ascending aorta injury by a displaced sternal plate through a multidisciplinary approach. Video available at: https:// www.jtcvs.org/article/S0022-5223(19)31722-2/fulltext.
This patient represented a challenge, particularly concerning the type of surgical approach and management. Reentry through the neosternum was initially considered too hazardous, and thus the first step involved total debranching of the brachiocephalic vessels, followed by placement of an endostent into the aortic arch covering the site of rupture. A total endovascular treatment was indeed considered unfavorable because of the presence of a bovine aortic arch. However, subsequent occurrence of an endoleak and stent compression by the plate mandated a second endostent insertion. Finally, the repair was completed by removing the sternal plate, avoiding the risk of a potentially catastrophic hemorrhage, and further sternal reconstruction.

We believe that the successful outcome in this patient can be attributed to the appropriate surgical strategy, as well as the involvement of vascular, thoracic, and plastic surgeons and interventional radiologists. This case demonstrates 
that with a multidisciplinary approach, even extremely challenging aortic pathologies can be managed successfully. Furthermore, it shows the need for close follow-up in patients after sternal reconstruction to detect this possible, albeit unusual, complication.

\section{References}

1. Nosotti M, Rosso L, Mendogni P, Tosi D, Palleschi A, Parafioriti A, et al. Sternal reconstruction for unusual chondrosarcoma: innovative technique. J Cardiothorac Surg. 2012;7:40.
2. Bongioloatti S, Voltolini L, Borgianni S, Borrelli R, Innocenti M, Menichini G et al. Short- and long-term results of sternectomy for sternal tumours. J Thorac Dis. 2017;9:4336-46.

3. Demondion P, Mercier O, Kolb F, Fadel E. Sternal replacement with a custommade titanium plate after resection of a solitary breast cancer metastasis. Interact Cardiovasc Thorac Surg. 2014;18:145-7.

4. Koto K, Sakabe T, Horie N, Ryu K, Murata H, Nakamura S, et al. Chondrosarcoma from the sternum: reconstruction with titanium mesh and a transverse rectus abdominis myocutaneous flap after subtotal sternal excision. Med Sci Monit. 2012;18:CS77-81.

5. Bertin F, Piccardo A, Denes E, Delepine G, Tricard J. Porous alumina ceramic sternum: a reliable option for sternal replacement. Ann Thorac Med. 2018;13:226-9. 